Mathematical Models for the

Study, Monitoring and Management

of the North Sea

Jacques C. J. Nihoul

Universities of Liège and Louvain and

Chairman of the Joint North Sea Modelling Group

A general interdisciplinary model of the North sea is presented. The model, which integrates the contributions of many neighbouring countries under the coordination of the Joint North Sea Modelling Group, feeds a hierarchy of submodels. These models,progressing from the description of transient and residual circulations to the dynamics of coastal ecosystems and the translocation of pollutants through the food chain,provide the material for continental seas and coastal management. Detailed results concerning, the Southern Bight of the North Sea and the Belgian Coastal waters are given in illustration.

\section{GLOSSARY OF SYMBOLS}

$U$ water transport vector (integral of velocity over deptn)

$h$ mean depth of the sea

5 surface elevation

$h=h+\zeta \quad$ total depth

f Coriolis frequency (twice the vertical component of the earth rotation vector)

$\xi$ astronomical tide-producing force per unit mass

$\mathrm{Pa}_{\mathrm{a}}$ atmospheric pressure

o specific mass of sea-water

$g$ acceleration of gravity

a turbulent and shear effect viscosity

$\mathrm{v}$ wind speed at anemometer level $(10 \mathrm{~m})$

D bottom friction coefficient

C sea surface drag coefficient

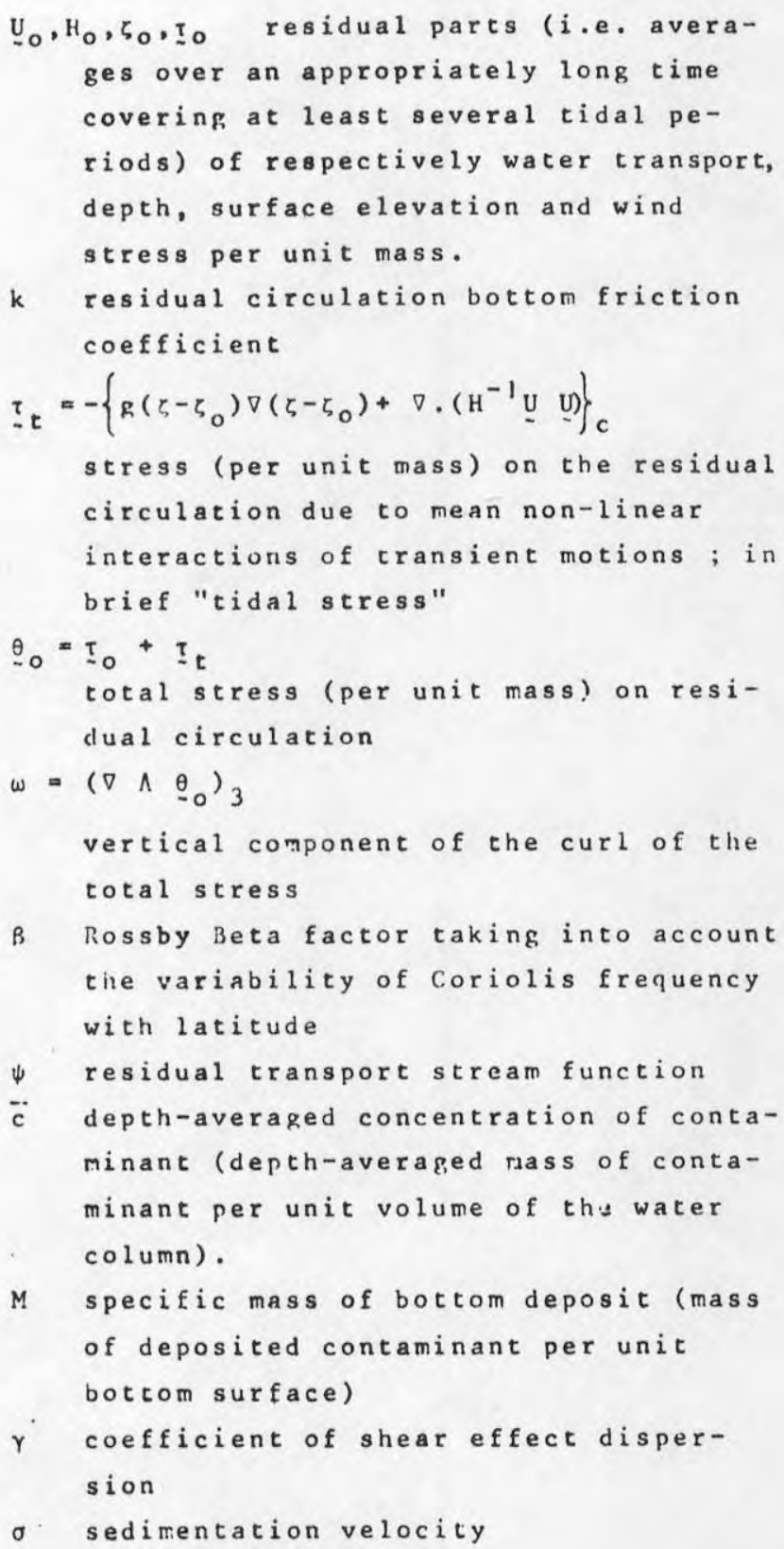


$U_{c}$ critical value of U above which disruption of the bottom layer by turbulence reverses the sedimentation flux and recirculates sediments in the water column

$\lambda$ turbulent diffusivity of contaminant multiplied by the total depth $\mathrm{H}$.

\section{INTRODUCTION}

Early mathematical models of the North Sea were primarily concerned with tides and storm surges predictions. The increased interest recently in fisheries management and pollution control has lead to spectacular development of mathematical modelling including residual circulation, dispersion of contaminants from coastal or off-shore releases, chemical and ecological dynamics. Although preliminary researchestended to address separately the Physics, the Chemistry or the Biology of the North Sea, it was soon realized that the development of computing facilities allowing more ambitious programs partial models could be progressively integrated in a general interdisciplinary model. This multipurpose research model seeking improved understanding of the North Sea environment was aimed at predicting the system's evolution under the constraints of Modern Society and providing the material for simpler, oriented "management" models - regarded as "subsets" of the general model - to answer specific questions with the degree of sophistication which the objectives, on the one hand, the reliability of data on the other hand, recommend.

In the same time, a group of coordination, the Joint North Sea Modelling Group, was set up to coordinate the national efforts of different countries bordering the North Sea. The organization of the international surveys of the North Sea (the Joint North Sea Data Acquisition Programs ; in brief "JONSDAP") provided the elements of a well documented data base and permanent interactions between models and observations, research and applications.

The application of systems analysis to the elaboration and calibration of the general model and its submodels, the data support and the mathematical and numerical forms of the models have been described and commented in earlier publications $(1,2,3)$.

In this paper, selection of new results is presented showing how, through a hierarchy of submodels, a predictive description of the North Sea system can be given from the transient and residual current patterns to the dynamics of coastal ecosystems and the translocation of pollutants though the food chain.

\section{THE MODELS}

Although it is conceptually important to work in the frame of a general interdisciplinary three-dimensional model, it is convenient to derive from it a sequence of complementary sub-models by which the marine system can be investigated progressively, starting from what is best known and can be modelled with the greatest reliability; and progressing, as data become available for models adjustments, to more sophisticated simulations

1. Jacques C.J.NIHOUL, Math Modelsea III, ICES Hydropraphy Committee CM 1974 C : 1, 1974.

2. Jacqiues C.J.NIHOUL, Modelzing of Marine Systems, Amsterdam : Elsevier, 1975.

3.Jacques C.J.NIHOUL, "Applicalion of Mathematical Models to the Scudy Monitoring and Management of the liorth Sea", in Ecological llodelling in a Resource Management Framework, Washington: Resources for the Future, 1975. 
and predictions. North Sea Modelling has thus proceeded from well-established hydrodynamic models to more conjectural ecosystems models attempting to assess the pathways of pollutants in the food chain.

(i) Integration over depth and reasonable hypotheses on the vertical density distribution have allowed the development of two dimensional models of tides and storm surges.

(ii) Further time integration (over a time sufficiently long to cover several tidal periods and thus cancel to a large extent tidal oscillations and transitory wind currents) has given the residual circulation model where the results of tidal computations are used to calculate the forcing due to non-linear tidal interactions.

(iii) Tidal and residual models have been exploited to evaluate the dispersion and the advection of marine constituents and to elaborate dispersion models adapted to the study of coastal discharges of pollutants and off-shore dumpings.

(iv) The hydrodynamic models have revealed distinctive marine regions where different current regimes prevail and which appear as natural boxes for the elaboration of completely space integrated time dependent chemical and ecological box models.

(v) By restricting attention to the aggregate properties of compartments such as dissolved substances, suspensions, bottom sediments, phytoplankton, zooplankton, heterotrophic bacteria, fish,.. pollution models have been developed to assess the pathways of pollutants in the food chain and evaluate the factors of concentration and the subsequent toxicity of marine food products.

A series of examples is given here illustrating the gradual insight one gets into the system with progressive emphasis on coastal waters off the Belgian coast.

a. Tide and Storm Surge Mode1

The equations governing long wave motions in the North Sea can be written (2) $\frac{\partial \mathrm{H}}{\partial t}+\nabla \cdot \underline{\mathrm{U}}=0$

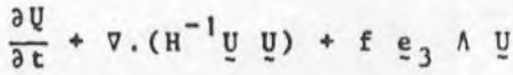

$=H\left(\xi-\nabla\left(\frac{P a}{\rho}+g \zeta\right)\right)+a \nabla^{2} \underline{U}$

$-\frac{\mathrm{D}}{\mathrm{H}^{2}} \mid \underline{\mathrm{u}}\|\underline{\mathrm{U}}+\mathrm{C} \underline{\mathrm{v}}\| \underline{\mathrm{v}} \|$

The notations are explained in the glossary .

The diffusivity a is of the order of $50 \mathrm{~m}^{2} / \mathrm{sec}$. The diffusive term can be estimated for long waves

a $\nabla^{2} \underline{U} \sim 50 \frac{10}{\left(210^{4}\right)^{2}} \sim 10^{-6} \mathrm{~m}^{2} \mathrm{sec}^{-2}$

This term is thus much smaller than the Coriolis, pressure and intertial terms $\left(10^{-4}\right.$ to $\left.10^{-3} \mathrm{~m}^{2} \mathrm{sec}^{-2}\right)$ and can be neglected.

The North Sea is 1 imited by coasts and by open-sea boundaries (straits of Dover, skagerrak, North Atlantic entrance). Appropriate boundary conditions along open sea boundaries are supplied by observations or by numerical predictions from other models covering more extended areas with coarser grids.

The current regime in the North Sea is mostly tidal. The most important partial tide is the lunar semi-diurnal. Coastal tidal data are well known and come from the Table deg Constantes Harmoniques de Monaco.

Currents and sea elevations are not purely harmonical function of time due to non linear terms in the equations of motion. These non linearitios aupear in shallow area; the Belgian, Dutch, German, Danish coasts are characterized by shallow waters. In order to compare the amplitude and phase of the $\gamma_{2}$ tide at coastal stations, a Fourier analysis of 
the sea level is carried out. The comparison between observed and calculated amplitudes and phases for coastal stations shows a very good agreement (4).

Fig. 1 and 2 show the cotidal and corange lines calculated by the model and derived from the observations of proudman and Doodson (5).

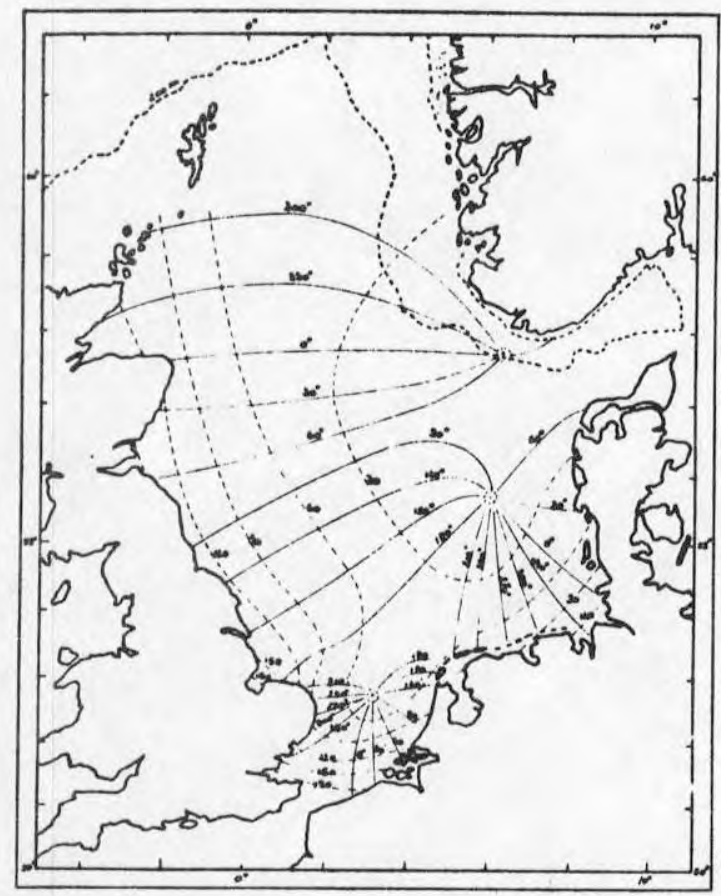

rig. 1. Lines of equal tidal phases and amplitudes in the North Sea according to the Mathematical Model.

4.François C.RONDAY, Ph.D. Dissertation, Liège University, 1975.

5.J. PROUDMAN and A.T. DOODSON, Philos. Trans. R. Soc. Lond. 224A, 185, 1924.

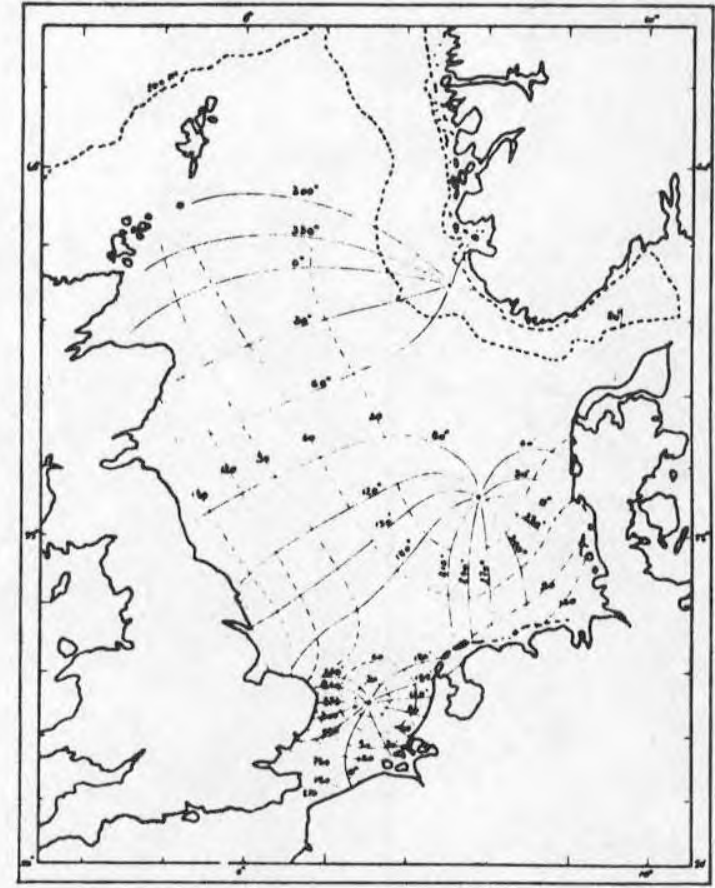

Fig. 2. Lines of equal tidal phases and amplitudes in the North Sea according to observations.

b. Residual Circulation Model

The long-term circulation in the North Sea is influenced by the North At lantic current which enters the North sea through the Straits of Dover and through the

Channel between the Orcades and Shetland Isles. The influence of the Baltic Sea is weak but provides water with a low content of salt $(34 \%)$. From oceanographic measurements $\left(\mathrm{T}, \mathrm{S}, \mathrm{O}_{2}\right)$ it is possible to determine the general distribution of the different water masses (6).

In winter the North Sea water has no haline and no thermal stratifications except in the Norwegian Channel and near the coast line. The barotropic model can be used. In summer the haline stratification remains weak but a thermal stratification exists in the northern and central

6.T. LAEVASTU, Serial Atzas of the Marine Environment, Washington D.C. Awer.

Geograph. Soc. 1963. 
part of the basin. The barotropic model however still provides fairly good results during that period of time.

The equations governing the residual circulation can be written (2

$f \stackrel{e}{\mathbf{e}} \wedge \stackrel{\text { U }}{0}$

$=\mathrm{H}_{0} \nabla\left\{\frac{\mathrm{Pa}_{\mathrm{a}}}{0}+\mathrm{g} \zeta\right\}_{0}-\frac{\mathrm{k}}{\mathrm{H}_{0}} \mathrm{U}_{0}+\underline{\theta}_{0}$

$\nabla \cdot \underline{U}_{0}=0$

or, eliminating the pressure and introducing a transport stream function

$k \nabla^{2} \psi-\frac{\partial \psi}{\partial x_{1}}\left(f \frac{\partial H_{0}}{\partial x_{2}}+\frac{2 k}{H_{0}} \frac{\partial H_{0}}{\partial x_{1}}-B\right)$

$+\frac{\partial \psi}{\partial x_{2}}\left(f \frac{\partial H_{o}}{\partial x_{1}}-\frac{2 k}{H_{0}} \frac{\partial H_{o}}{\partial x_{2}}\right)$

$=H_{0} \omega+\frac{\partial H_{0}}{\partial x_{2}} \theta_{1}-\frac{\partial H_{0}}{\partial x_{1}} \theta_{2}$

The notations are defined in the glossary. $e_{3}$ is the unit vector along the vertical axis pointing, upwards. $\theta_{1}$ and $\theta_{2}$ the component of $\theta_{0}$ along the horizontal axes $\sim_{1}$ and $e_{2}$ pointing to the east and to the north respectively.

The boundary conditions on eq. (5) are derived from observations of water in and out - flows accross open sea boundaries and inflows from the main rivers (4). Along the coasts, the condition

$\psi$ = constant is imposed.

The total stress ${\underset{\sim}{0}}_{0}$ is calculated from the mean wind stress over the period of interest $\left(\tau_{0} \sim 210^{-5} \mathrm{~m}^{2} / \mathrm{sec}^{2}\right.$, for the North Sea) and the "tidal stress" computed using the results of the preliminary long waves model.

One of the original features of the residual model described here is the explicit introduction in the residual equations of the residuary effect of the nonlinear interactions of transient motions.

To emphasize the importance of this effect, the residual equations were first solved without explicitly taking the tidal stress into account. The residual circulation pattern so obtained - although it reproduced roughly the distribution of water masses inferred from ti.e observations - fails to uncover a series of secondary gyres along. the liritish and Belgian coasts, around the Dopger bank and in the German Bight; the existence of which has been demonstrated by long series of currentmeters data (7). This is empiasized in figs. (3) and (4) showing the resilual circulation in t..e Southern Bight pridicted by the classical model (without tidal stress) and by tre new model (incorporating explicitly the tidal stress).

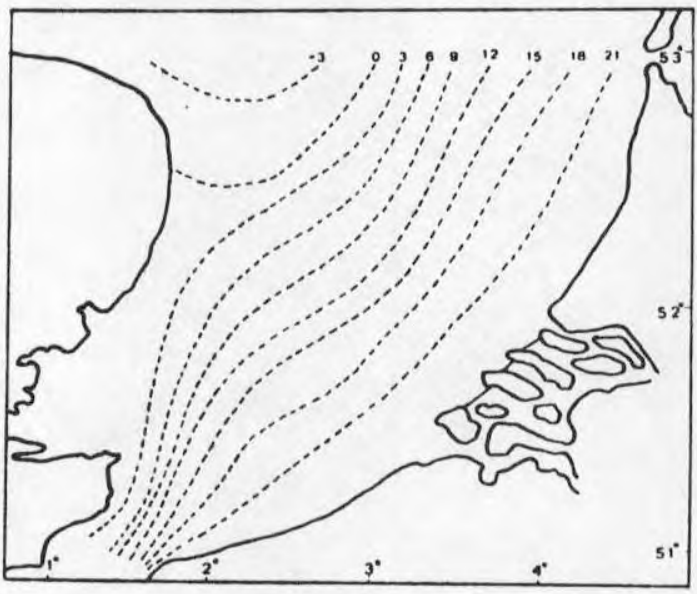

Fig. 3. Residual Circulation in the Southern Bight wit:out Lidal stress.

streamlines $\psi$ const (in $\left.10^{4} \mathrm{~m}^{3} / \mathrm{s}\right)$.

The model with the tidal stress predicts the existence of a residual gyre off the Belgian coast with a south westerly current along the coast. Current observations confirm the existence of the gyre which also explains the distribution of sediments in the Bight and in particular the presence of a surprisinoly large

7.H.W. HILL, Current and Water Masses, North Sea Science, MIT University Press, p. 17-42. 
area of mud deposition in front of the Northern Belgian coast (8). The gyre is responsible for partially entraining the highly turbid Scheldt water to the South West and for increasing the residence time of water masses in the area with only very low escape possibilities of the sediments to the North.

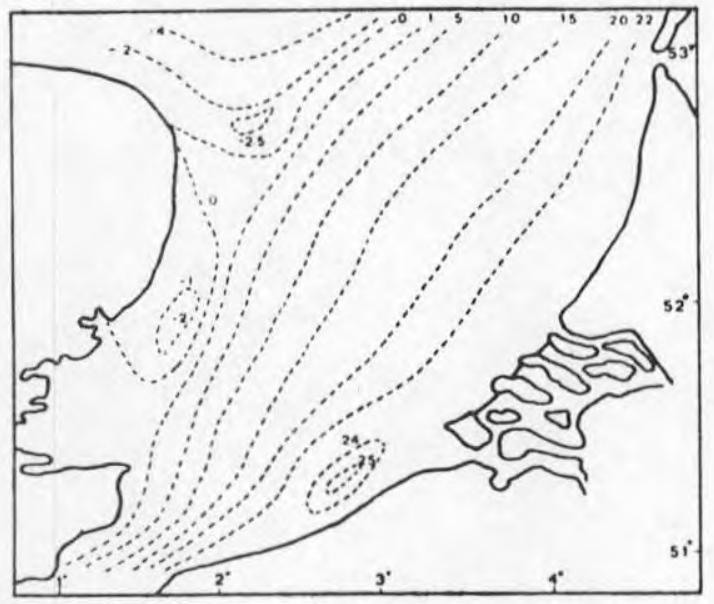

Fig. 4. Residual Circulation in the Southern Bight with the tidal stress.

Streamlines $\psi$ aconst (in $10^{4} \mathrm{~m}^{3} / \mathrm{s}$ ).

\section{c. Dispersion models}

The depth-averaged equations describing the dispersion in the sea of a marine contaminant taking into account the eventual effects of sedimentation and bottom erosion, can be written (2)

II $\frac{\partial \ddot{c}}{\partial t}+\underline{U} \cdot \nabla \bar{c}$

$$
\begin{aligned}
& =\nabla \cdot H\left\{\gamma \frac{\underline{U}}{U}(\underline{U}, \nabla \bar{c})\right\} \\
& -\sigma \bar{c}\left(1-\frac{U^{2}}{U_{c}^{2}}+\nabla \cdot(\lambda \nabla \bar{c})\right.
\end{aligned}
$$

४.J.C.J.NIlioul, Effect of the tidal stress on mud deposition in the southern Bight of the North Sea, Proc. 2nd Annual Meeting of the European Geophysical Societu, Trieste, 20-26 sept. 1974. $\frac{\partial M}{\partial t}=\sigma \bar{c}\left(1-\frac{U^{2}}{U_{c}^{2}}\right)$

The notations are defined in the glossary. The equation describing the evolution of the mean concentration of suspensions is derived from the three-dimensional dispersion equation by integrating over depth. The average over depth of the quadratic advection terms gives two contributions : the first one contains the product of the means, the seconc one the mean product of the deviatione around the means.

The structure of the latter is analo. pous to that of the Reynolds stress and experiments reveal that it is indeed responsible for an enhanced dispersion comparable to - but often more important than - the turbulent dispersion. This effect is called the "shear effect" becaur se it is associated with the existence of a vertical velocity gradient.

The shear effect has been described by several authors in pipes, cuannels and estuaries where, after integration over the cross section, the flow - steady or oscillating - is essentially in one direction.

In the shallow waters of the Southern Bight, it is penerally sufficiant to consider the mean concentrations over the depth but, out at sea, no further averaging. is possible and the dispersion mechanism is fundamentally two-dimensional. A generalized model was thus developed to account for the rotation of the tidal velocity vector and also include the sedimentation and partial recirculation of the solid suspensions $(2,9)$.

9.J.C.J.NIHOLL, Diffusion of turbidity by shear effect and turhulence in the Southern Bipht of the :"orth Sea, Aävances in Geophysics, 18 A, :.331-337, 1974 . 


\section{d. Chemical-ecological Models}

An extensive survey of chemical and ecological variables in the Eastern part of the Southern Bight was made during the years 1971, 1972, 1973 and 1974 in the scope of the Belgian National Program on the Environment, Sea Project. On the basis of these informations, the survey region was divided into three zones where different conditions prevail. These three zones were found to correspond to different residual circulation regimes as predicted by the model.

(i) The first zone, off the Belgian coast, corresponds to the residual gyre. Water from the scheldt estuary is to a large extent entrained in the gyre before it can escape to the North. An island of high turbidity ( $\left.10^{-2} \mathrm{~kg} \mathrm{~m}^{-3}\right)$ is observed in the region where closed stream lines are predicted by the model. Bottom sediments in this zone are characterized by large areas of mud which can be explained by the tendency to form - as shown by the residual current model - an outerlagoon in which increased suspended mater arrival tends to be preserved by the current pattern, is flocculated and aggregated by biological activity and preserved from net erosion by weakened wave activity.

(ii) The central zone corresponds to fairly parallel stream lines showing off the north-bound flow of the branch of the Gulf Stream which penetrates the North Sea through the Straits of Dover. The turbidity in this zone is considerably lower and no systematic silt deposition occurs.

(iii) The third zone, off the Dutch Coast, corresponds to water masses under direct influence of the Meuse-Rhine estuary. The rivers' outfall is in a sense prolonged into the sea by a residual flow parallel to the Dutch coast, to the North.
The lagoon conditions which result from the residual circulation in zone 1 is responsible for striking differences between the dynamics of the ecosystems in that region and in zone 2 .

The high turbidity of tus water in zone 1 causes light extinctior and reduces primary productivity. The dominance of microplankton in that refion (as compared to nannoplankton in zone 2) with a higher half saturation constant could affect productivity in the sare way. Taking into account, also, that the depth is smaller in the coastal repion, one should expect the inteprated production (over depth) to be considerably smaller in zone 1. Observations reveal however that - although there are differences in the annual variation - the yearly average is about the same in the two regions (e.g. 10).

This result is obviously related to the much higher nutrient concentration and the much larger specific phytoplankton biomass (measured by chloroshyll a) observed in zone 1 where lagoon conditions prevail $($ e.g. 10).

Additional information are provided by the ratio phaeophytin a / chlorophyll a which is systematically larger than one in zone 1 and smaller than one in zone 2 ; indicating that, in zone $1, \operatorname{most}$ of the phytoplankton cells are dead cells. Zooplankton grazing, on the other hand, is more important in zone 2 than in zone 1 , where planktonic and benthic heterotrophic bacteria seem to play the essential role.

10.J.C.J.NIHOUL, Mesoscale secondary flows. Implications in the chemical and biochemical dynamics of the Southern Bight of the North Sea, Proc. Sth Liège Colloquium on Ocean Hydrodynamics, Liège April 18 May 2, 1974. 
The dynamics of the ecosystems thus reflects the residual circulation patterns and the outerlagoon situation created by the residual gyre in zone 1.

\section{e. Pollution Mode1s}

By revealing the existence of distinctive regions with different circulation patterns and subsequent water residence times, variform sediments distribution and chemical concentrations in the water column and distinct ecosystems dynamics induced to some extent by diversified physical and chemical characteristics and diverse coastal and off-shore man-imposed new constraints, the hierarchy of models identifies the natural boxes for adjacent box models describing the dynamics of the ecosystems. These models can evaluate the fluxes of carbon, nitrogen,... pollutants through the food web and provide estimates of the fish population and its level of pollution. Such models - although still conjectural in some aspects - have been successfully developed and compare satisfactorily with the observations (1). They provide the framework for the ultimate management of continental seas $(2,3)$ and require the priority attention of the new generation of marine scientists.

\section{CONCLUSIONS}

The general interdisciplinary model of the North Sea and its hierarchy of submodels can assist marine management. hydrodynamic models can predict with great accuracy the elevation of the water surface produced by tides and storm surges, the transient and residual current patterns, the dispersion, sedimentation and eventual recirculation by strong turbulence - of suspended material as well as the final deposition of sediments on the bottom.
Their results can be exploited to assess the effects of coastal engineering. works (dredging, construction of harbours and artificial island, location of epuration units...).

(ii) Dispersion models can evaluate the extent of the dammage produced by a given coastal or off-shore release and appreciate the opportunity of authorizing or penalizing sea dumpings.

(iii) Chemical and ecolorical rodels can elaborate on hydrodynamic and dispersion models to construct the transfer functions which relate the intensity of tie sources of pollution, the subsequent curcentrations in the sea and the final accumulation in the food chain.

The model can then assist public Health decisions or working backwards from Public tolerances, set up for manasement the problem of optimizing the nounts and locations of tolerable pollution inputs subject to the constraints of Mociern society. 